\title{
Mitteilungen
}

\section{Schweizerische Gesellschaft für Angiologie}

\section{Schweizerischer Angiologiepreis 2012}

Die Schweizerische Gesellschaft für Angiologie verleiht einen Preis von 10000 CHF für eine wissenschaftliche Arbeit über Gefässkrankheiten. Die Arbeit kann ein zur Publikation eingereichtes Manuskript oder bereits veröffentlicht sein (2011-2012).

Curriculum Vitae und Bewerbung für den Angiologiepreis sind bis zum 31. August 2012 in je 5 Exemplaren einzusenden an: Prof. Henri Bounameaux, Hôpitaux Universitaires de Genève (HUG), Service d'angiologie et d'hémostase, 2 , rue Gabrielle-Perret-Gentil, 1211 Genève 14.

Der Preis wird anlässlich der 13. Unionstagung 21.-23. November 2012 in Bern verliehen.

\section{Schweizerische Akademie der Medizinischen Wissenschaften SAMW}

\section{Ausschreibungen in den Bereichen Versorgungsforschung und Neurowissen- schaften}

Die Schweizerische Akademie der Medizinischen Wissenschaften schreibt für 2012 den Théodore-Ott-Preis in Neurowissenschaften (Preis für ein Lebenswerk) sowie den RobertBing-Preis für junge Forschende in Neurowissenschaften aus. Bewerbungsschluss für beide Preise ist der 31. August 2012.

Zudem hat die zweite Ausschreibungsrunde des neuen Förderprogramms «Versorgungsforschung im Gesundheitswesen» begonnen. Einsendeschluss für Gesuche ist der 1. September 2012.

Weitere Informationen zu den Ausschreibungen finden Sie auf der SAMW-Website www. samw.ch/de/Aktuell/Ausschreibungen.html

\section{Schweizerische Gesellschaft für Thoraxchirurgie SGT}

\section{Preis der SGT}

Die Schweizerische Gesellschaft für Thoraxchirurgie vergibt einen Preis von 5000 Franken für die beste publizierte Arbeit aus dem Gebiet der Thoraxchirurgie. Die Arbeit muss in der Schweiz entstanden sein und von Ende Mai 2011 bis Ende Juni 2012 publiziert oder zur Publikation angenommen sein. Die Überreichung und Veröffentlichung erfolgt an der SGT-Tagung am 15. November 2012 in Bern. Die Arbeiten sind bis zum 19. September 2012 einzureichen per Mail im PDF-Format an: r.inderbitzi[at]thoraxzentrum.ch (PD Dr. Rolf Inderbitzi, Sekretär und Vizepräsident der SGT, Thoraxzentrum Zürich, Facharzt FMH für Chirurgie, Witellikerstrasse 40, 8032, Zürich).

\section{Schweizerische Gesellschaft für Nuklearmedizin SGNM}

\section{Vorstand per 1.6.2012}

Präsident und Kongresspräsident 2012

Prof. G. K. von Schulthess, Zürich

Vizepräsident

Prof. T.M. Krause, Bern

Kassier

Dr. R. Oettli, St. Gallen

Sekretär

Dr. M. Wissmeyer, Genf, La Chaux-de-Fonds

Kongresspräsident 2013

PD Dr. K. Strobel, Luzern

Beisitzer

PD Dr. L. Giovanella, Bellinzona, Lugano

Prof. P.A. Kaufmann, Zürich

Prof. J. O. Prior, Lausanne

Prof. D. Wild, Basel
Schweizerische Gesellschaft

für Kardiologie SGK /

Société Suisse de Cardiologie SSC

\section{Vorstand 2012/2013}

Präsident/Président

Prof. Dr. med. François Mach, Genève

Pastpräsident

Prof. Dr. med. Hans Rickli, St. Gallen

Vizepräsident/Vice-président

President-Elect

Prof. Dr. med. Georg Noll, Zürich

Kassier/Trésorier

Dr. med. Dominé Frédéric, Biasca

Vertreter Kinderkardiologie/Représentant Cardiologie pédiatrique

Prof. Dr. med. Jean-Pierre Pfammatter, Bern

Mitglieder/Membres

Prof. Dr. med. Christine Attenhofer Jost, Zürich

Dr. med. Jacqueline Landolt, Morges

Dr. med. Urs Kaufmann, Bern

Dr. med. Patrick Monnier, La Chaux-de-Fonds Prof. Dr. Jürg Schwitter, Lausanne

Prof. Dr. med. Stephan Windecker, Bern

Prof. Dr. med. Michael Zellweger, Basel

Geschäftsführerin/Directrice

Dr. med. Marjam Rüdiger-Stürchler, St. Gallen

Sekretariat/Secrétariat

Karin Guldenfels, Bern 


\section{Obsan}

Die Ausgaben der obligatorischen Krankenpflegeversicherung sind in den zwölf Jahren von 1998 bis 2010 teuerungsbereinigt um 6,9 Milliarden Franken gestiegen. Die Zunahme lässt sich nur zu einem kleinen Teil dem Bevölkerungswachstum oder der Alterung zuordnen. Es sind vor allem die ambulanten Behandlungen sowie die Medikamente, die zu deutlich höheren Kosten führten. Dies zeigt eine Mehrjahres-Studie des Schweizerischen Gesundheitsobservatoriums (Obsan).

Die Gesamtausgaben im Gesundheitswesen der Schweiz beliefen sich 2010 auf 62,5 Milliarden Franken. Knapp ein Drittel dieser Ausgaben 22 Milliarden Franken, wurde durch die obligatorische Krankenpflegeversicherung (OKP) gedeckt. Zwischen den Jahren 1998 und 2010 haben die Ausgaben im Rahmen der OKP um jährlich 4,6 Prozent zugenommen, stärker als die Gesamtausgaben $(+3,8 \%)$. Dies zeigt sich deutlich bei den Pro-Kopf-Kosten, welche miteinander verglichen wurden. Während diese Kosten im Jahr 1998 für eine versicherte Person durchschnittlich 1640 Franken betrugen, waren es 2010 rund 2680 Franken - unter Berücksichtigung der Teuerung eine Zunahme um 3,2 Prozent pro Jahr.

Drei Leistungsbereiche der Krankenversicherungen decken 92 Prozent der Gesamtausgaben ab: die ambulante Behandlung, die stationäre Behandlung sowie die Vergütung von Medikamenten und Material. Am stärksten nahmen die Kosten von 1998 bis 2010 bei Medikamenten und Material zu ( $+4,9 \%$ pro Jahr). Auch die Kosten im Bereich der ambulanten Behandlung stiegen deutlich an $(+4,2 \%$ pro Jahr).

Die stationäre Behandlung in Spitälern zeigte eine vergleichsweise moderate Kostenzunahme $(+3,3 \%$ pro Jahr). Insbesondere haben neue therapeutische Methoden dazu geführt, dass die Betroffenen kürzer stationär oder oft auch ambulant behandelt werden können. Spitalaufenthalte wurden in den letzten Jahren trotzdem teurer, da während des Aufenthalts mehr oder teurere Leistungen angeboten werden.

Je nach Kanton fallen die OKP-Gesamtkosten unterschiedlich aus. Die Analyse des Obsan zeigt, dass Kantone, welche 1998 überdurch- schnittlich hohe Pro-Kopf-Kosten hatten, fast durchwegs auch 2010 überdurchschnittlich hohe Kosten aufwiesen. Die höchsten Pro-KopfKosten zeigten über den gesamten Zeitraum die Kantone Genf und Basel-Stadt; die tiefsten die Kantone Appenzell Innerrhoden und Nidwalden, was auf strukturelle Unterschiede der Kantone hindeutet. Auffallend ist auch, dass die Kantone Genf und Waadt einen deutlich tieferen Anteil der Ausgaben im stationären Bereich aufweisen, der durch höhere Ausgaben für ambulante Leistungen kompensiert wird.

Alterung und Bevölkerungszunahme erklären nur einen Fünftel der Kostenveränderung zwischen 1998 und 2010. Wichtigste Ursache des Kostenzuwachses bei den ambulanten Leistungen im Spital ist die Zunahme der Konsultationen. Bei den in der Arztpraxis behandelnden Spezialistinnen und Spezialisten ist der Kostenzuwachs darauf zurückzuführen, dass die Konsultationen von älteren Personen zugenommen haben und dass das Leistungsvolumen für Letztere gestiegen ist. Zusätzlich spielt die Wahl neuerer und teurerer Medikamente eine Rolle, und zu einem kleineren Teil auch die Zunahme des Pro-Kopf-Konsums.

Das Obsan geht davon aus, dass die OKP-Kosten auch in den kommenden Jahren weiter zunehmen werden. Zum einen wird die Alterung der Bevölkerung zu einer Zunahme chronischer und damit kostenintensiver Krankheiten führen. Zum anderen werden bedeutende technische Fortschritte erwartet, die zwar die Qualität der Behandlung verbessern, aber auch zu einer weiteren Kostenzunahme beitragen werden.

\section{Schweizerische Stiftung SPO Patientenschutz}

\section{Patientenschutz fordert Beweislastumkehr für Versuchspersonen}

Die Patientenschützerin und St. Galler Nationalrätin Margrit Kessler fordert beim Bundesrat eine Beweislastumkehr für Versuchspersonen der medizinischen Forschung. Ohne erleichterte Beweisführung haben es Patienten ausserordentlich schwer, Schadenersatzansprüche für gesundheitliche Schäden im Rahmen von klinischen Studien durchzusetzen.
Die SPO sieht sich immer wieder mit Fällen konfrontiert, bei denen Patienten im Rahmen eines Forschungsprojekts nicht nur einen gesundheitlichen Schaden erleiden, sondern auch die finanziellen Konsequenzen davon tragen müssen. Der entsprechende Gesetzesartikel im Schweizer Heilmittelgesetz (HMG) bietet für solche Fälle leider keinen ausreichenden Schutz. Heute hat der Geschädigte den umfassenden Nachweis zu erbringen, dass der Arzneimittelversuch den Gesundheitsschaden verursachte. Diese patientenfeindliche Rechtslage hat der Bundesrat im vergangenen Mai in seiner Antwort auf eine Interpellation der SPO-Präsidentin und St. Galler Nationalrätin Margrit Kessler bestätigt. Beweislasterleichterungen oder eine Beweislastumkehr zugunsten der Patienten lehnte die Landesregierung in ihrer Interpellationsantwort ab [1]. Weil die Probanden durch das geltende Schweizer Recht nur ungenügend geschützt werden, rät die SPO derzeit von der Teilnahme an solchen Forschungsprojekten ab. Jetzt fordert Margrit Kessler in einer soeben eingereichten Motion eine Änderung der entsprechenden Bestimmung im Heilmittelgesetz [2]. 31 Mitglieder des Nationalrats haben den Vorstoss mit unterzeichnet.

Bei einer Beweislastumkehr müssten Sponsoren von klinischen Tests wie zum Beispiel Pharmafirmen beziehungsweise die Haftpflichtversicherungen nachweisen, dass der Schaden bei der Versuchsperson nicht auf den Einsatz des Studienmedikaments zurückzuführen ist, sondern auch ohne die Teilnahme am Forschungsprojekt eingetreten wäre. In Deutschland habe sich die Beweislastumkehr bei der Haftung für Arzneimittelschäden bewährt, erklärt Patientenschützerin Margrit Kessler.

1 www.parlament.ch/d/suche/seiten/geschaefte. aspx?gesch_id $=20123222$

2 www.parlament.ch/d/suche/seiten/geschaefte. aspx?gesch_id=20123452 